\title{
Effectiveness and safety of roxadustat in the treatment of anemia of kidney disease: a systematic review and meta-analysis
}

\author{
Hui Wang ${ }^{1}$, Ke Huang ${ }^{2}$, Chun Wang ${ }^{3}$, Chunyan Chen ${ }^{1}$, Hui Fang ${ }^{1}$, Jin Cao ${ }^{1}$ \\ ${ }^{1}$ Department of Nephrology, China Coast Guard Hospital of the People's Armed Police Force, Jiaxing, China; ${ }^{2}$ Department of Nephrology, Zhejiang \\ Chinese Medical University Affiliated Jiaxing TCM Hospital, Jiaxing, China; ${ }^{3}$ Department of Clinical Laboratory, China Coast Guard Hospital of \\ the People's Armed Police Force, Jiaxing, China \\ Contributions: (I) Conception and design: H Wang, J Cao; (II) Administrative support: J Cao; (III) Provision of study materials or patients: H Wang, \\ K Huang, C Wang; (IV) Collection and assembly of data: All authors; (V) Data analysis and interpretation: K Huang; (VI) Manuscript writing: All \\ authors; (VII) Final approval of manuscript: All authors. \\ Correspondence to: Jin Cao. Department of Nephrology, China Coast Guard Hospital of the People's Armed Police Force, 16 Nanhu Road, Nanhu \\ District, Jiaxing 314000, China. Email: 13656831122@163.com.
}

Background Chronic kidney disease is a global public health problem, anemia is the most common complication of chronic kidney disease. Roxadustat is a new drug that can be used to treat renal anemia by oral administration.

Methods: We conducted a comprehensive computerized search of the China Knowledge Network (CNKI), VIP (VIP), Wanfang, China Biomedical (CBM), PubMed, Embase, and the Cochrane Library databases. We collected clinical randomized controlled trials (RCTs) published on roxadustat in the treatment of anemia of kidney disease. According to the inclusion and exclusion criteria, the included studies were subject to screening and quality evaluation, and RevMan 5.3 software was used for meta-analysis.

Results: A total of 7 RCTs involving 997 patients with anemia of kidney disease were included. The results of meta-analysis showed that compared with the treatment effect of the erythropoietin (EPO) group, in the roxadustat group there were significant increases in the amount of hemoglobin [mean difference (MD) $=11.70,95 \%$ confidence interval $(\mathrm{CI}): 0.41$ to $22.99, \mathrm{P}=0.04$ ], hemoglobin overall response rate [odds ratio $(\mathrm{OR})=1.97,95 \% \mathrm{CI}: 1.7$ to $3.33, \mathrm{P}=0.01$ ], serum iron [standardized mean difference $(\mathrm{SMD})=0.39,95 \% \mathrm{CI}$ : 0.23 to $0.55, \mathrm{P}<0.00001$ ], transferrin saturation $(\mathrm{MD}=6.09,95 \% \mathrm{CI}: 5.36$ to $6.82, \mathrm{P}<0.0001)$, transferrin $(\mathrm{MD}=0.36,95 \% \mathrm{CI}: 0.28$ to $0.44, \mathrm{P}<0.00001]$, total iron binding capacity ( $\mathrm{SMD}=0.63,95 \% \mathrm{CI}: 0.47$ to 0.79 , $\mathrm{P}<0.00001)$ and significantly reduced hepcidin ( $\mathrm{MD}=-29.04,95 \% \mathrm{CI}:-51.61$ to $-6.47, \mathrm{P}=0.01)$. There was no significant difference in adverse reactions between groups (OR $=1.06,95 \%$ CI: 0.30 to 3.74, $\mathrm{P}=0.93)$.

Discussion: Roxadustat can effectively improve the anemia status of patients with CKD without increasing adverse reactions. Due to limitations of the number and quality of the included studies, further verification is required through the multi-center, large sample-sized, and high-quality RCTs in the future.

Keywordsः Roxadustat; chronic kidney disease (CKD); anemia; meta-analysis

Submitted Jan 27, 2021. Accepted for publication Apr 21, 2021.

doi: $10.21037 /$ apm-21-456

View this article at: http://dx.doi.org/10.21037/apm-21-456

\section{Introduction}

Anemia is considered one of the most common concomitances associated with chronic kidney disease (CKD) $(1,2)$ and is related to a higher incidence of cardiovascular disease, mortality, and morbidity along with the poorer quality of life and prognosis (3). Based on evidence of the improvement of the anemia status of 50 $70 \%$ of patients with CKD (4), the current recommendation for clinical management of anemia in CKD is erythropoietic stimulants combined with iron supplements $(5,6)$. 
However, erythropoietic stimulants usually need to be administered by subcutaneous injection and cause pain and discomfort; additionally, delivery of iron requires regular hospitalization for intravenous supplementation. Finally, patient compliance in the treatment process cannot be ensured, leading to different outcomes of the treatment for anemia of kidney disease. With the arrival of orally active inhibitors of hypoxia-inducible factor (HIF) prolyl hydroxylase, roxadustat has gained much scientific interest in the treatment of anemia in CKD (7). However, roxadustat is still poorly characterized for efficacy and safety in anemia of kidney disease due to insufficient time on the market and inconsistent research results. Our meta-analysis therefore aimed to systematically review the efficacy and safety of roxadustat for anemia in patients with kidney disease, providing substantial evidence for further clinical application of drugs in the management of anemia of kidney disease.

We present the following article in accordance with the PRISMA reporting checklist (available at http://dx.doi. org/10.21037/apm-21-456).

\section{Methods}

\section{Criteria for inclusion and exclusion}

\section{Study eligibility criteria}

We included randomized controlled trials (RCTs) with or without the blind method. Studies were restricted to those published in Chinese and English.

\section{Objects}

We reviewed studies involving participants who met the diagnostic criteria of anemia of kidney disease (8), with no restrictions on age, gender, and course of disease.

\section{Intervention}

The experimental group was treated with roxadustat, and the control group was treated with erythropoietin (EPO), with no restrictions on the total daily dose and course of treatment.

\section{Outcome measures}

(I) Hemoglobin level; (II) total hemoglobin reaction rate; (III) serum iron; (IV) transferrin saturation; (V) transferrin; (VI) total iron binding capacity; (VII) iron modulator; (VIII) adverse events (AEs).

\section{Exclusion criteria}

(I) Non-RCT study; (II) studies published in non-Chinese and non-English languages; (III) duplicate studies, experience summaries, case studies, reviews, studies with incomplete outcome data, animal experiments, meeting minutes; (IV) studies evaluating less than 15 cases; (V) studies on anemia of reasons other than kidney disease, such as primary anemia and hemorrhagic anemia; (VI) studies only involving individuals treated by roxadustat alone, without control measures; (VII) studies with participants who failed to take medicine regularly and quantitatively as prescribed by the doctor; (VIII) attrition of participants was $>20 \%$.

\section{Search strategy}

We searched studies through the China National Knowledge Internet (CNKI), the VIP, Wanfang, Chinese Biomedical Database (CBM), MEDLINE (PubMed), and Embase databases, and The Cochrane Library from inception to 20 November 2020 using Chinese and English search terms relevant to this review. Chinese search terms used were roxadustat, FG-4592, CKD, chronic renal failure (CRF), and anemia. English search terms used were roxadustat, kidney failure, chronic kidney disease, CKD, kidney disease, and chronic renal failure.

\section{Data extraction and quality assessment}

\section{Data extraction}

General information was independently extracted by 2 of the authors (e.g., the first author, year of publication, research period, disease state, the number of cases, age, intervention, and treatment), outcome indicators (total $\mathrm{Hb}$ response rate, $\mathrm{Hb}$, serum iron, transferrin saturation, transferrin, total iron binding capacity, iron element, AEs, and so on). Disagreement was resolved by a third investigator. When relevant data was missing or unclear, attempts were made to contact the original author for complete data, and we excluded studies without such data available.

\section{Quality assessment}

Studies that presented during the search were independently appraised by two reviewers according to the inclusion and exclusion criteria. In the case of any disagreements or discrepancies were resolved through consultation with 
a third reviewer. The overall quality of studies included was evaluated by the improved Jadad rating scale (9). The scoring includes 4 aspects: (I) generation of random sequences; (II) allocation concealment; (III) blind method; (IV) follow up (withdrawal and quit). Scores of 1-3 points indicated low quality studies, and scores of 4-7 points indicated high quality studies.

\section{Data analysis}

The RevMan software package (Review Manager, version 5.3, The Nordic Cochrane Center, The Cochrane Collaboration, 2014) was used for meta-analyses and statistical tests. The mean difference (MD) or standardized mean difference (SMD) was used for measurement of continuous variables. Odds ratio (OR) was used as the effect analysis statistic, and interval estimation was presented with $95 \%$ confidence interval (CI). The assessment of heterogeneity between the included studies was performed by $\chi^{2}$ test. When there was no significant heterogeneity between the results $\left(\mathrm{P}>0.10, \mathrm{I}^{2} \leq 50 \%\right)$, the fixed effects model was used for meta-analysis; otherwise, the random effects model was used. Repeated operation analysis was performed to estimate the same index. One study was deleted each time to show the impact of a particular study on the combined effect, and then the study was excluded for sensitivity analysis. At the same time, the heterogeneity between subgroups was assessed when there was heterogeneity. A P value $<0.05$ was considered statistically significant.

\section{Results}

\section{Characteristics of articles}

A total of 286 studies were gathered from the initial search. After selection of studies according to the inclusion and exclusion criteria, 7 studies were eligible for final analysis (10-16); a total of 997 patients were included, 588 of whom were in the experimental group and 409 were in the control group. The whole study selection process is depicted in Figure 1. The basic information of studies is shown in Table 1.

\section{Methodological quality evaluation results}

Among 7 RCTs, 4 were of high quality (1 scored 7 marks,
3 scored 4 marks). The remaining 3 studies were of low quality (3 scored 3 marks) (Table 2).

\section{Meta-analysis report}

\section{Hemoglobin}

The improvement of hemoglobin in patients with anemia of kidney disease treated with roxadustat was reported in 4 studies (10,14-16) (497 patients). Significant heterogeneity was found among studies $\left(\mathrm{P}<0.00001, \mathrm{I}^{2}=95 \%\right)$. The fixed effect model combined effect size was used for analysis. With treatment of roxadustat, hemoglobin was significantly higher than that of the EPO group $(M D=11.70$, 95\% CI: 0.41 to $22.99, \mathrm{P}=0.04$ ) (Figure 2). Sub-group analysis (Chinese and non-Chinese) was based on racial categorization. No heterogeneity was found among Chinese studies (14-16) $\left(\mathrm{P}=0.95, \mathrm{I}^{2}=0 \%\right)$. The fixed effect model combined effect size was used for analysis. With treatment of roxadustat, hemoglobin was significantly higher than that of the EPO group (MD =16.50, 95\% CI: 13.06 to 19.95, $\mathrm{P}<0.00001$ ) (Figure 3). A qualitative description was made for the studies that were excluded in this meta-analysis. Akizawa et al. conducted an RCT in 2020 including 301 patients, with 150 receiving roxadustat and 151 receiving EPO (10). With treatment of roxadustat, hemoglobin was significantly higher than that of the EPO group ( $\mathrm{P}=0.03)$ (Figure 3).

\section{Total hemoglobin response rate}

The total hemoglobin response rate in patients with anemia of kidney disease treated with roxadustat was assessed in 4 studies $(11-13,16)$ (556 patients). No significant heterogeneity was found among studies $\left(\mathrm{P}=0.28, \mathrm{I}^{2}=21 \%\right)$. The fixed effect model combined effect size was used for analysis. With treatment of roxadustat, the total hemoglobin response rate was significantly higher than that of the EPO group ( $\mathrm{OR}=1.97,95 \% \mathrm{CI}: 1.7$ to $3.33, \mathrm{P}=0.01)$ (Figure 4).

\section{Serum iron}

The improvement of serum iron amplitude in patients with anemia of kidney disease treated with roxadustat was reported in 3 studies $(10,12,13)$ (638 patients). No significant heterogeneity was found among studies $(\mathrm{P}=0.67$, $\left.\mathrm{I}^{2}=0 \%\right)$. The fixed effect model was used for analysis. With treatment of roxadustat, the serum iron amplitude was significantly higher than that of the EPO group (SMD $=0.39,95 \%$ CI: 0.23 to $0.55, \mathrm{P}<0.00001$ ) (Figure 5). 


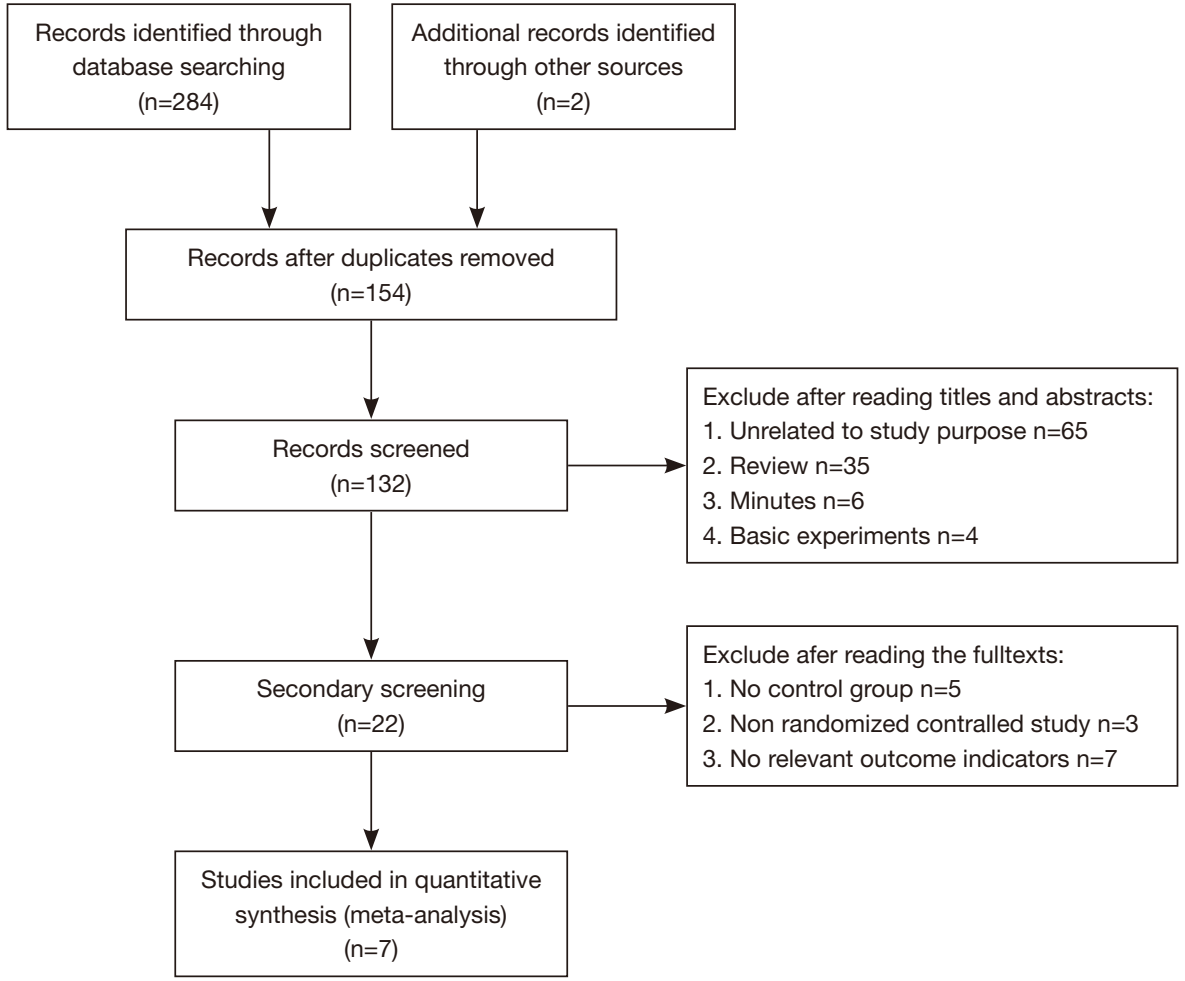

Figure 1 Study selection.

Table 1 General information of included studies

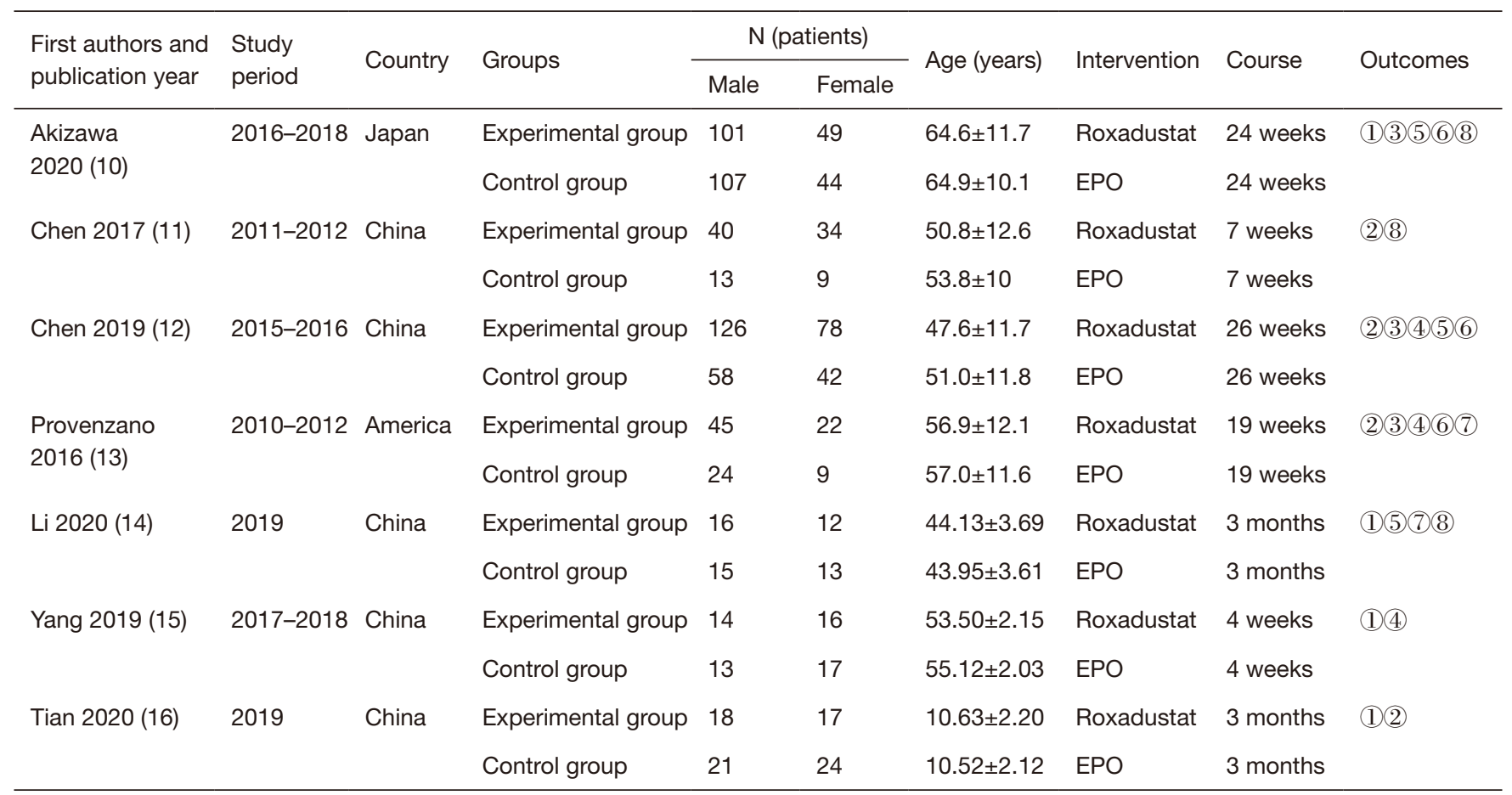

(1) Hemoglobin; (2) total hemoglobin reaction rate; (3) serum iron: (4) transferrin saturation: (5) transferrin: (6) total iron binding capacity;

(7) hepcidin: (8) adverse events. 
Table 2 Methodological quality evaluations of included studies

\begin{tabular}{|c|c|c|c|c|c|}
\hline First authors and publication year & Random method & Allocation concealment & Blind method & Follow up & Scores \\
\hline Chen 2017 (11) & 2 & 0 & 1 & 1 & 4 \\
\hline Chen 2019 (12) & 2 & 0 & 1 & 1 & 4 \\
\hline Provenzano 2016 (13) & 2 & 0 & 1 & 1 & 4 \\
\hline Yang 2019 (15) & 2 & 0 & 0 & 1 & 3 \\
\hline Tian 2020 (16) & 2 & 0 & 0 & 1 & 3 \\
\hline
\end{tabular}

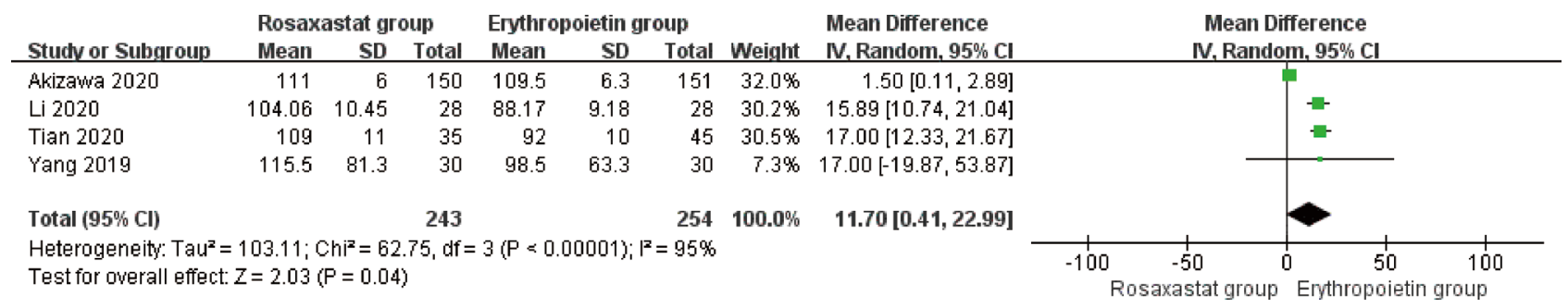

Figure 2 Forest plot of meta-analysis of hemoglobin after treatment in two groups.

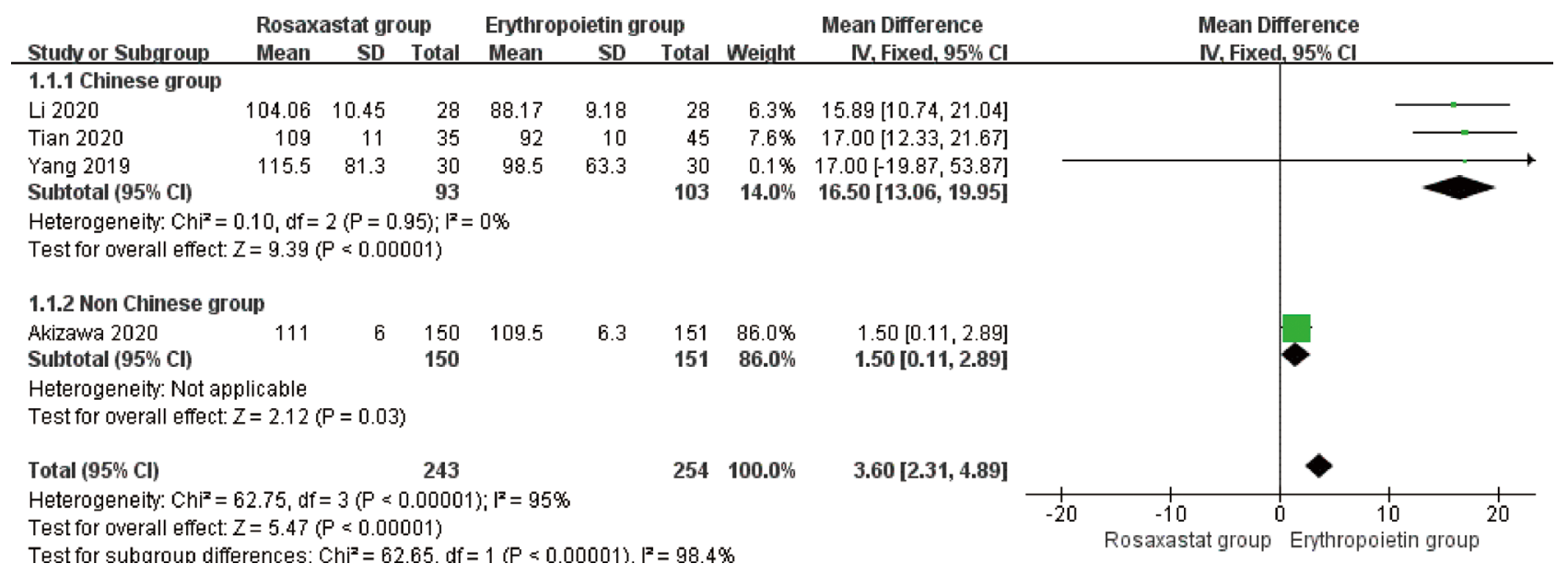

Figure 3 Forest plot of meta-analysis of hemoglobin subgroups after treatment of different races in two groups.

\section{Transferrin saturation}

The improvement of transferrin saturation in patients with anemia of kidney disease treated with roxadustat was reported in 3 studies $(12,13,15)$ (397 patients). No significant heterogeneity was found among studies $(\mathrm{P}=0.55$, $\left.\mathrm{I}^{2}=0 \%\right)$. The fixed effect model combined effect size was used for analysis. With treatment of roxadustat, transferrin saturation was significantly higher than that of the EPO group $(\mathrm{MD}=6.09,95 \% \mathrm{CI}: 5.36$ to $6.82, \mathrm{P}<0.0001)$ (Figure 6).

\section{Transferrin}

The improvement of transferrin in patients with anemia of kidney disease treated with roxadustat was reported 


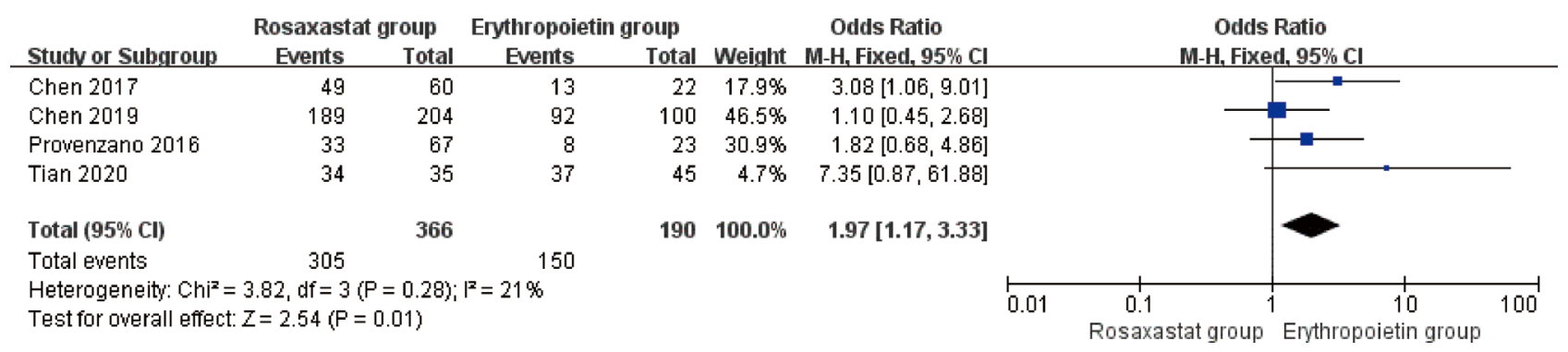

Figure 4 Forest plot of meta-analysis of hemoglobin overall response rate in two groups.

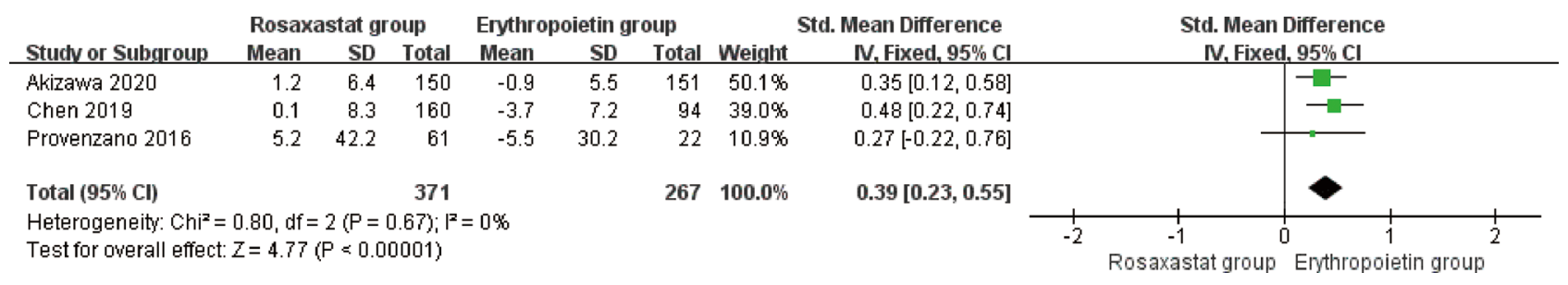

Figure 5 Forest plot of meta-analysis of the range of serum iron changes in two groups.

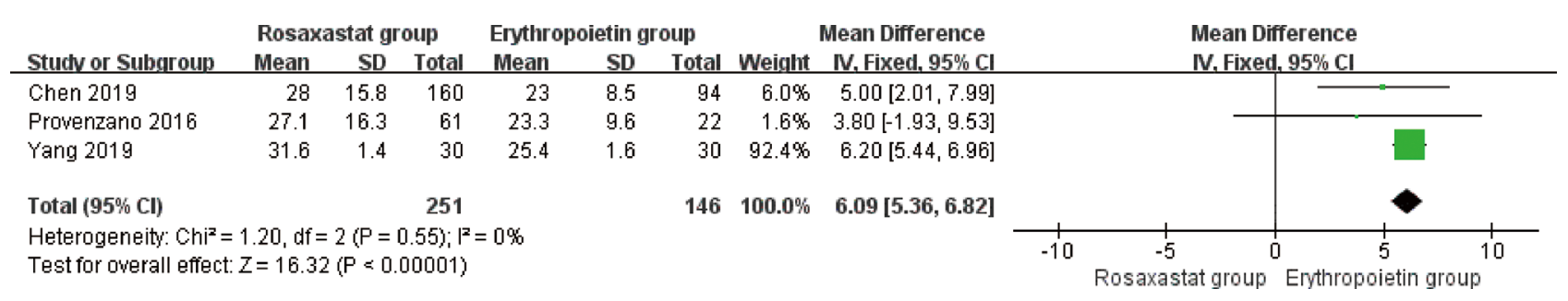

Figure 6 Forest plot of meta-analysis of percentage of transferrin saturation in two groups.

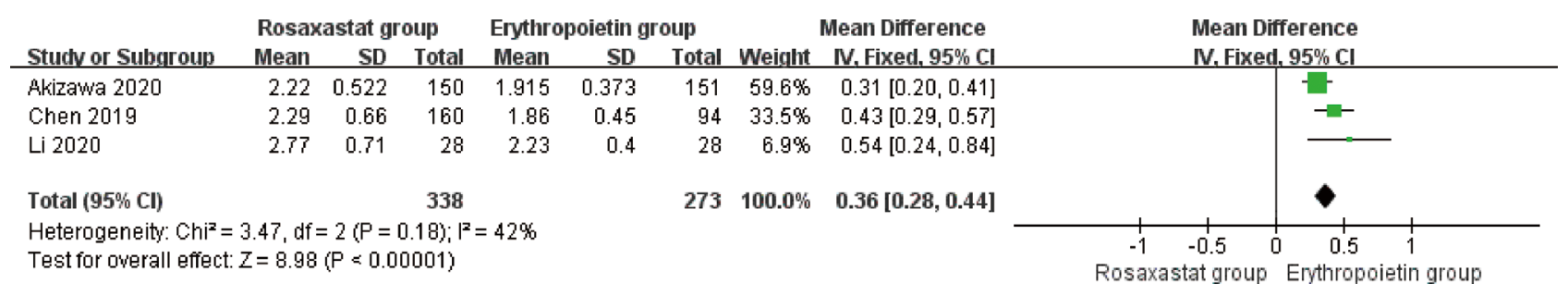

Figure 7 Forest plot of meta-analysis of transferrin in two groups.

in 3 studies $(10,12,14)$ (611 patients). No significant heterogeneity was found among studies $\left(\mathrm{P}=0.18, \mathrm{I}^{2}=42 \%\right)$. The fixed effect model combined effect size was used for analysis. With treatment of roxadustat, transferrin was significantly higher than that of the EPO group ( $\mathrm{MD}=0.36$, 95\% CI: 0.28 to $0.44, \mathrm{P}<0.00001$ ) (Figure 7).

\section{Total iron binding ability}

The improvement of total iron binding ability in patients with anemia of kidney disease treated with roxadustat was as reported in 3 studies $(10,12,13)$ (638 patients). No significant heterogeneity was found among studies $\left(\mathrm{P}=0.15, \mathrm{I}^{2}=47 \%\right)$. The fixed effect model combined effect size was used for 


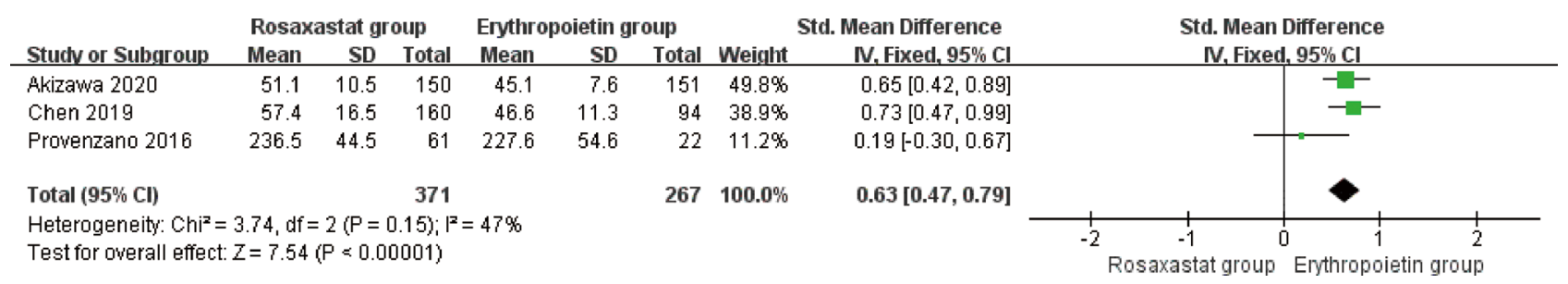

Figure 8 Forest plot of meta-analysis of total iron-binding capacity in two groups.

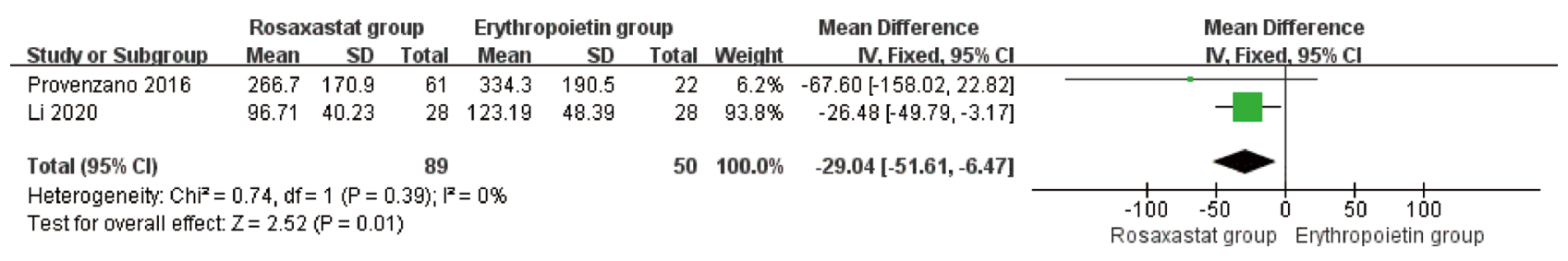

Figure 9 Forest plot of meta-analysis of hepcidin in two groups.

analysis. With treatment of roxadustat, the total iron binding ability was significantly higher than that of the EPO group (SMD $=0.63,95 \%$ CI: 0.47 to $0.79, \mathrm{P}<0.00001$ ] (Figure 8 ).

\section{Hepcidin}

The reduction of hepcidin in patients with anemia of kidney disease treated with roxadustat was reported in 2 studies $(13,14)$ (139 patients). No significant heterogeneity was found among studies $\left(\mathrm{P}=0.39, \mathrm{I}^{2}=0 \%\right)$. The fixed effect model combined effect size was used for analysis. With treatment of roxadustat, hepcidin was significantly more than that of the EPO group (MD $=-29.04,95 \%$ CI: -51.61 to $-6.47, \mathrm{P}=0.01$ ) (Figure 9).

\section{AEs}

The most common AEs included infections/infestations, gastrointestinal disorders, injury, poisoning, and procedural complications. The AEs may occurred in anemia of kidney disease patients included gastroenteritis cellulitis, diarrhea, diabetic gangrene, endocarditis bacterial, pneumonia, sepsis, gangrene, metabolism or nutrition disorder, fluid overload, hyperkalemia, diabetic ketoacidosis hypocalcemia, hypokalemia, acute myocardial infarction, cardiac disorder, cardiac failure congestive, cardiorespiratory arrest, gastrointestinal hemorrhage, gastrointestinal disorder, pancreatitis acutea, nausea, vomiting, injury, poisoning, or procedural complication, arteriovenous fistula site hemorrhage, shunt stenosis, contusion, vascular disorder, vascular graft complication, nervous system disorder, cerebrovascular accident, complex partial seizures, chronic obstructive pulmonary disease, pulmonary edema, sudden cardiac death, hypersensitivity, musculoskeletal chest pain, thyroid neoplasm, major depression, subcutaneous emphysema, muscle spasms, diarrhoea, abdominal discomfort, dizziness, headache, immune system disorder, hypertension, hepatobiliary disorder, decreased appetite, transferrin saturation (TSAT) decreased, renal or urinary disorder, renal failure chronic, nasopharyngitis, upper respiratory tract infections, chest discomfort, asthenia, alanine aminotransferase increased, hypotension, blood or lymphatic system disorder, endocrine disorder, product issue, reproductive system or breast disorder. Among them, the incidence of roxadustat in terms of infection, myocardial infarction, stroke, heart failure, and death were differed in every RCT trial and presented in Table S1.

The AEs in patients with anemia of kidney disease treated with roxadustat was reported in 3 studies $(10,11,14)$ (454 patients). Significant heterogeneity was found among studies $\left(\mathrm{P}=0.01, \mathrm{I}^{2}=77 \%\right)$. The fixed effect model combined effect size was used for analysis. There was no statistically significant difference in the incidence of AEs between the two groups ( $\mathrm{OR}=1.06,95 \% \mathrm{CI}$ : 0.30 to $3.74, \mathrm{P}=0.93$ ) (Figure 10). Sensitivity analysis showed that after excluding the studies sequentially, there was no directional change in the combined effect value, indicating that results of this study were basically stable. 


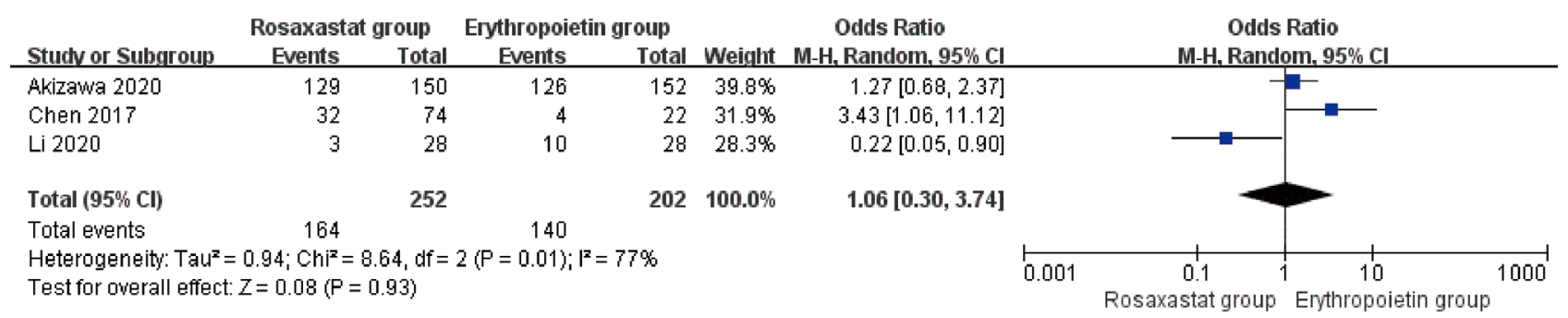

Figure 10 Forest plot of meta-analysis of adverse reactions in two groups.

\section{Publication bias}

Since there were only relatively few studies, a funnel plot for publication bias was not applied in this meta-analysis.

\section{Discussion}

The pathogenesis mechanism of anemia of kidney disease is reasonably complex. Patients with renal failure and disorders of iron metabolism often present with relative or absolute diminished EPO and iron absorption (17) which subsequently leads to anemia (18). Although dialysis is recognized as a life-saving measure for CKD, with this treatment the disease can ultimately progress into anemia. Hemoglobin deficiency not only worsens CKD but also increases risk of cardiovascular disease and death $(19,20)$. Besides, severity of the anemia and CKD is associated with hospitalization and complications (21). Thus, clinical management of anemia of kidney disease is of marked importance. Injectable erythropoiesis-stimulating agents (ESAs) and iron agents are the traditional treatments for anemia in CKD; however, this standard care is challenged by various issues, for example, the treatment can be easily affected by inflammation, iron absorption, EPO antibodies, and regular and quantitative medication. Also, traditional treatments increase the risk of hypertension, cardiovascular disease, oxidative stress, and allergic reactions (22). Therefore, the need remains for improved treatment of anemia in CKD. In recent decades, efforts have been made to minimize risk while maximizing therapeutic benefits.

In a series of intriguing studies on roxadustat, inhibition of small molecule preserved ammonia acyl hydroxylase inhibitor (HIF-PHI), which is an erythropoietic response during hypoxia, was noted. HIF is a heterodimeric transcription factor responsible for the hypoxic induction of erythropoietin (EPO) and other oxygen-sensitive genes. As the first oral drug for the treatment of anemia of kidney disease (23), roxadustat significantly inhibits HIF-HIF-
PHI, stabilizing the $\alpha$ subunit of HIF-1; additionally, downstream target-gene-encoding proteins, such as heme biosynthase, EPO, EPO receptor, and proteins associated with iron absorption and transport are affected. Consequently, erythropoiesis is promoted by these proteins through regulation of EPO and iron. The backbone of conducting roxadustat therapy treatment is to maintain the hemoglobin $(\mathrm{Hb})$ levels within $10-12 \mathrm{~g} / \mathrm{dL}$. In previous investigations, we already know that roxadustat increased $\mathrm{Hb}$ levels in the manner of dose-dependently and the halflife of roxadustat is $8-12 \mathrm{~h}$. Chen et al. investigated the starting dose of roxadustat by clarifying into two groups 1.0 and $1.5 \mathrm{mg} / \mathrm{kg}$ (12). Chen et al. concluded that starting doses at about $1.5 \mathrm{mg} / \mathrm{kg}$ were needed to take into account initial $\mathrm{Hb}$ level response during the period of fixed-dose and the number of dose adjustments required after 4 weeks. The roxadustat dose for $\mathrm{Hb}$ level maintenance ranged from 0.5 to 3.4 (mean dose, 1.7 ) $\mathrm{mg} / \mathrm{kg}$ thrice weekly while the roxadustat dose was not to exceed $3 \mathrm{mg} / \mathrm{kg}$ or $300 \mathrm{mg}$ (12).

In our analysis, a total of 7 RCTs and 997 participants with anemia of kidney disease were included. We found roxadustat was associated with better treatment response in terms of the improvement of hemoglobin, total hemoglobin response rate, serum iron, transferrin saturation, transferrin and total iron binding ability, and reduction of ferritin between the roxadustat group when compared with the EPO group.

Provenzano et al. show that roxadustat can increase the level of endogenous erythropoietin to within or near the physiological range and improve iron homeostasis. The level of endogenous erythropoietin was increased to the physiological range $(20.6-29.1 \mathrm{mIU} / \mathrm{mL})$ at about $6 \mathrm{~h}$ since roxadustat was treated. And there is a peak point near $140 \mathrm{mIU} / \mathrm{mL}$ of endogenous erythropoietin level at about $12 \mathrm{~h}$ since roxadustat treated before it decreased. At about $24 \mathrm{~h}$ after roxadustat treated, the level of endogenous erythropoietin decreased to the physiological range again 
and was maintained in the range for more hours than its climb.

In almost all of the studies ( 5 in 7, 2 of them didn't measure hepcidin), a progressive decrease in hepcidin levels was observed at higher doses of roxadustat treated patients (10-14). This indicated that roxadustat can regulated iron homeostasis by reduced hepcidin levels.

Because roxadustat binds to potently inhibits hypoxiainducible factor-prolyl hydroxylase (HIF-PHD), reducing HIF breakdown and promoting its activity. HIF-PHD enzymes regulate the stability of the HIF- $\alpha$ subunit of the HIF transcription factor by post-translational hydroxylation of HIF in an oxygen-dependent manner, thus maintaining a balance between oxygen availability and HIF activity. The central role of HIF-PHD enzymes as gatekeepers of coordinated transcriptional adaptation to hypoxia and oxidative stress makes them attractive therapeutic targets for the treatment of anemia. Inhibition of HIF-PHD enzymes leads to the accumulation of functional HIF, an increase in endogenous EPO production (resulting in enhanced erythropoiesis), and indirect suppression of hepcidin.

In the comparison of roxadustat $v s$. EPO, no difference was observed for AEs, indicating that roxadustat was effective and safe for treatment of anemia. The use of roxadustat is expected to be a more convenient (oral) and safer option for the management of anemia of kidney disease. However, most of the included studies did not use double-blind or concealment strategy, which means further investigations are needed to more accurately estimate the effects.

The studies have reported that the frequency of hyperkalemia and upper respiratory tract infections in the roxadustat group is higher. We consider the reason for this is the different criteria among the trial sites. An adverse reaction is defined as an adverse event that is considered to be related or possibly related to the trial drug by the investigator. Trial sites had different criteria for the reporting of hyperkalemia as an adverse event during treatment. Trend analyses from baseline to the end of the trial did not show any meaningful increase in potassium levels. Considering the underlying disease (chronic kidney disease), the causal relationship between the adverse reaction of hyperkalemia and roxadustat is unclear. Although it is possible that the reporting of hyperkalemia might reflect a potential bias inherent in the trial comparing roxadustat with placebo in patients with chronic kidney disease not undergoing dialysis, hyperkalemia and metabolic acidosis were reported more frequently in the roxadustat group. The intermittent central laboratory monitoring may not have detected potassium elevations, and therefore continued evaluation will be important in presently ongoing trials and as wider experience with roxadustat occurs. Nasopharyngitis is a common adverse event of roxadustat treatment. Due to the short-term observation of the studies and the main focus on the efficacy of roxadustat, it is not powered to provide a definitive conclusion on the influence of upper respiratory tract infections. Trial sites had different criteria for the reporting of nasopharyngitis as an adverse event during treatment. In this case, this report is consistent with previous results. However, further investigation will be required to solve this problem.

Five of the seven trials record short-term use of roxadustat increased hemoglobin levels in anemic patients with chronic kidney disease. Two of the seven trials conduct the long-term investigation of roxadustat for more than 6 months. The long-term efficacy is similar between roxadustat treated patients and epoetin alfa treated patients. Roxadustat displays a manageable safety profile but more treated patients have adverse events which may due to the previous design of the studies. Further investigation of the safety will be required to firmly establish the safety profile of roxadustat.

Our analysis of the 7 RCT shows a consistent conclusion that the baseline inflammatory, indicating by CRP level didn't influence the effect of roxadustat on increasing $\mathrm{Hb}$.

Roxadustat therapy treatment improved the quality of life in the following aspects. First, roxadustat could reduce hepcidin, lower plasma cholesterol while didn't increase platelet levels. Second, the convenience of oral administration for roxadustat is supported by the high treatment compliance, reducing the need for frequent hospital visits. Third, oral iron therapy provided results equivalent to those of intravenous iron therapy with roxadustat whereas oral iron is expected to be ineffective relative to intravenous iron with epoetin alfa.

Although inclusion and exclusion criteria were strictly observed in this meta-analysis, a few limitations persisted, such as the heterogeneity led by insufficient evidence, limitations in sample size, and differences in the duration of intervention among studies. According to the methodological details of the study design, the standardization of RCT research needs to be strengthened: (I) some studies failed to attach great importance to the status of allocation concealment and blindness in RCTs; (II) some studies did not record the reasons for cases of loss to follow-up and withdrawal. 
In conclusion, the administration of roxadustat appeared to be an effective strategy to improve anemia in CKD without increasing AEs. Nevertheless, large sample sized, multi-centered RCTs of high quality, strict design, and long-term follow-up are still needed for verification of our findings.

\section{Acknowledgments}

Funding: Zhejiang Medical Association Clinical Research Fund Project (ZYC-A95 2018).

\section{Footnote}

Reporting Checklist: The authors have completed the PRISMA reporting checklist. Available at http://dx.doi. org/10.21037/apm-21-456

Conflicts of Interest: All authors have completed the ICMJE uniform disclosure form (available at http://dx.doi. org/10.21037/apm-21-456). The authors have no conflicts of interest to declare.

Ethical Statement: The authors are accountable for all aspects of the work in ensuring that questions related to the accuracy or integrity of any part of the work are appropriately investigated and resolved.

Open Access Statement: This is an Open Access article distributed in accordance with the Creative Commons Attribution-NonCommercial-NoDerivs 4.0 International License (CC BY-NC-ND 4.0), which permits the noncommercial replication and distribution of the article with the strict proviso that no changes or edits are made and the original work is properly cited (including links to both the formal publication through the relevant DOI and the license). See: https://creativecommons.org/licenses/by-nc-nd/4.0/.

\section{References}

1. Atkinson MA, Warady BA. Anemia in chronic kidney disease. Pediatr Nephrol 2018;33:227-38.

2. Nakhoul G, Simon JF. Anemia of chronic kidney disease: Treat it, but not too aggressively. Cleve Clin J Med 2016;83:613-24.

3. Kaiafa G, Kanellos I, Savopoulos C, et al. Is anemia a new cardiovascular risk factor? Int J Cardiol 2015;186:117-24.

4. UK renal registry 15 th annual report: appendix e methodology for estimating catchment populations of renal centres in England for dialysis patients. Nephron 2013;123:213-5.

5. Zeidan A, Bhandari S. Anemia in peritoneal dialysis patients: iron repletion, current and future therapies. Perit Dial Int 2017;37:6-13.

6. Ford D, Gilg J, Williams AJ, et al. UK renal registry 19th annual report: chapter 7 haemoglobin, ferritin and erythropoietin amongst UK adult dialysis patients in 2015: national and centre-specific analyses. Nephron 2017;137:165-88.

7. Joharapurkar AA, Pandya VB, Patel VJ, et al. Prolyl Hydroxylase Inhibitors: A Breakthrough in the Therapy of Anemia Associated with Chronic Diseases. J Med Chem 2018;61:6964-82.

8. Mikhail A, Shrivastava R, Richardson D. Renal Association Clinical Practice Guideline on anaemia of chronic kidney disease. Nephron Clin Pract 2011;118:c101-c124.

9. Jadad AR, Moore RA, Carroll D, et al. Assessing the quality of reports of randomized clinical trials: is blinding necessary? Control Clin Trials 1996;17:1-12.

10. Akizawa T, Iwasaki M, Yamaguchi $Y$, et al. Phase 3, Randomized, Double-Blind, Active-Comparator (Darbepoetin Alfa) Study of Oral Roxadustat in CKD Patients with Anemia on Hemodialysis in Japan. J Am Soc Nephrol 2020;31:1628-39.

11. Chen N, Qian J, Chen J, et al. Phase 2 studies of oral hypoxia-inducible factor prolyl hydroxylase inhibitor FG4592 for treatment of anemia in China. Nephrol Dial Transplant 2017;32:1373-86.

12. Chen N, Hao C, Liu BC, et al. Roxadustat Treatment for Anemia in Patients Undergoing Long-Term Dialysis. N Engl J Med 2019;381:1011-22.

13. Provenzano R, Besarab A, Wright S, et al. Roxadustat (FG-4592) Versus Epoetin Alfa for Anemia in Patients Receiving Maintenance Hemodialysis: A Phase 2, Randomized, 6- to 19-Week, Open-Label, ActiveComparator, Dose-Ranging, Safety and Exploratory Efficacy Study. Am J Kidney Dis 2016;67:912-24.

14. Li H, Liang J, Ma Q. Comparison of efficacy and prognosis of Roxadustat Capsules and recombinant human erythropoietin in the treatment renal anemia. China Medical Herald 2020;17:178-81.

15. Yang J. The effect of losartan in patients with renal anemia and its influence on micro inflammatory factors. Health must-Read Magazine 2019;26:199-200.

16. Tian X, Tian X, Li F. Effect of losartan on renal anemia in children. Maternal and Child Health Care of China 
2020;35:3415-7.

17. Ahluwalia N, Skikne BS, Savin V, et al. Markers of masked iron deficiency and effectiveness of EPO therapy in chronic renal failure. Am J Kidney Dis 1997;30:532-41.

18. Wu CJ, Chen CY, Lai TS, et al. The role of indoxyl sulfate in renal anemia in patients with chronic kidney disease. Oncotarget 2017;8:83030-7.

19. Babitt JL, Lin HY. Mechanisms of anemia in CKD. J Am Soc Nephrol 2012;23:1631-4.

20. Eriksson D, Goldsmith D, Teitsson S, et al. Crosssectional survey in CKD patients across Europe describing the association between quality of life and anaemia. BMC
Nephrol 2016;17:97.

21. Ebben JP, Gilbertson DT, Foley RN, et al. Hemoglobin level variability: associations with comorbidity, intercurrent events, and hospitalizations. Clin J Am Soc Nephrol 2006;1:1205-10.

22. Wen S, Yang L. Standardized treatment of chronic renal anemia. Clinical Focus 2016;31:636-40.

23. Locatelli F, Fishbane S, Block GA, et al. Targeting HypoxiaInducible Factors for the Treatment of Anemia in Chronic Kidney Disease Patients. Am J Nephrol 2017;45:187-99.

(English Language Editor: J. Jones)

Cite this article as: Wang H, Huang K, Wang C, Chen C, Fang H, Cao J. Effectiveness and safety of roxadustat in the treatment of anemia of kidney disease: a systematic review and meta-analysis. Ann Palliat Med 2021;10(4):4736-4746. doi: 10.21037/apm-21-456 
Supplementary

Table S1 Incidence of Roxadustat in adverse events

\begin{tabular}{lccccc}
\hline & $\begin{array}{c}\text { Roxadustat treated } \\
\text { patients number }\end{array}$ & Infection & Myocardial infarction & Stroke & Heart failure \\
\hline Provenzano R 2016 & 108 & $8(7.4)$ & $2(1.9)$ & $2(1.9)$ & $1(0.9)$ \\
Chen N 2017 & 121 & $7(5.8)$ & - & - & - \\
Chen N 2019 & 204 & $42(20.6)$ & - & - & - \\
Akizawa T 2020 & 150 & $67(44.7)$ & $1(0.7)$ & - & $-1(0.7)$ \\
Li 2020 & 28 & - & - & - \\
\hline
\end{tabular}

Data are presented as $\mathrm{N}(\%)$. No mention is presented as -. 